DEMONSTRATIO MATHEMATICA

Vol. XLII No $4 \quad 2009$

\title{
Aurelian Cernea
}

\section{ON A BOUNDARY VALUE PROBLEM FOR A THIRD ORDER DIFFERENTIAL INCLUSION}

\begin{abstract}
We consider a boundary value problem for third order nonconvex differential inclusion and we obtain some existence results by using the set-valued contraction principle.
\end{abstract}

\section{Introduction}

This paper is concerned with the following boundary value problem

$$
x^{\prime \prime \prime}+k^{2} x^{\prime} \in F(t, x), \quad \text { a.e. }([-1,1]), \quad x(-1)=x(1)=x^{\prime}(1)=0
$$

where $F(.,):.[-1,1] \times \mathbb{R} \rightarrow \mathcal{P}(\mathbb{R})$ is a set-valued map and $k \in[-\pi, \pi]$.

The present note is motivated by a recent paper of Bartuzel and Fryszkowski ([1]), where it is considered problem (1.1) and a version of the Filippov Lemma for this problem is obtained. The aim of our paper is to present two additional results obtained by the application of the set-valued contraction principle due to Covitz and Nadler ([10]).

The first result follows a classical idea by applying the set-valued contraction principle in the space of solutions of the problem. The second result is also a Filippov type theorem concerning the existence of solutions to problem (1.1). Recall that for a differential inclusion defined by a Lipschitzian set-valued map with nonconvex values, Filippov's theorem consists in proving the existence of a solution starting from a given "quasi" solution. This time we apply the contraction principle in the space of derivatives of solutions instead of the space of solutions. In addition, as usual at a Filippov existence type theorem, we obtain an estimate between the starting "quasi" solution and the solution of the differential inclusion. The idea of applying the set-valued contraction principle in the space of derivatives of the solutions belongs to Bressan, Cellina and Fryszkowski ([2]) and it was used for

2000 Mathematics Subject Classification: 34A60, 26 A24.

Key words and phrases: boundary value problem, differential inclusion, contractive set-valued map, fixed point. 
the first time by Tallos $([11,13])$ in deriving Filippov type results. Other similar results concerning differential inclusions may be found in [4-9] etc..

The Filppov type result we propose in our approach is an alternative to the one in [1]. The two results are not comparable since the hypotheses concerning the quasi solution are different. Moreover, the methods used in their proofs are also different: the proof of the result in [1] follows Filippov's construction, while in our approach we obtain a "pointwise" estimate from a norm estimate.

For the motivation of the study of problem (1.1) we refer to [1] and references therein.

The paper is organized as follows: in Section 2 we recall some preliminary facts that we need in the sequel and in Section 3 we prove our main results.

\section{Preliminaries}

In this short section we sum up some basic facts that we are going to use later.

Let $(X, d)$ be a metric space and consider a set valued map $T$ on $X$ with nonempty values in $X . T$ is said to be a $\lambda$-contraction if there exists $0<\lambda<1$ such that:

$$
d_{H}(T(x), T(y)) \leq \lambda d(x, y) \quad \forall x, y \in X,
$$

where $d_{H}(.,$.$) denotes the Pompeiu-Hausdorff distance. Recall that the$ Pompeiu-Hausdorff distance of the closed subsets $A, B \subset X$ is defined by

$$
d_{H}(A, B)=\max \left\{d^{*}(A, B), d^{*}(B, A)\right\}, \quad d^{*}(A, B)=\sup \{d(a, B) ; a \in A\},
$$

where $d(x, B)=\inf _{y \in B} d(x, y)$.

The set-valued contraction principle ([10]) states that if $X$ is complete, and $T: X \rightarrow \mathcal{P}(X)$ is a set valued contraction with nonempty closed values, then $T($.$) has a fixed point, i.e. a point z \in X$ such that $z \in T(z)$.

We denote by $F i x(T)$ the set of all fixed points of the set-valued map $T$. Obviously, $\operatorname{Fix}(T)$ is closed.

Proposition 2.1. ([12]) Let $X$ be a complete metric space and suppose that $T_{1}, T_{2}$ are $\lambda$-contractions with closed values in $X$. Then

$$
d_{H}\left(F i x\left(T_{1}\right), F i x\left(T_{2}\right)\right) \leq \frac{1}{1-\lambda} \sup _{z \in X} d\left(T_{1}(z), T_{2}(z)\right) .
$$

Let $I=[-1,1]$. By a solution of problem (1.1) we mean a function $x(.) \in W:=W^{1,3}(I) \cap H_{0}^{1}(I)$ satisfying (1.1).

As usual, we denote by $C(I, \mathbb{R})$ the Banach space of all continuous functions from $I$ to $\mathbb{R}$ with the norm $\|x(.)\|_{C}=\sup _{t \in I}|x(t)|$ and $L^{1}(I, \mathbb{R})$ the Banach space of integrable functions $u():. I \rightarrow \mathbb{R}$ endowed with the norm $\|u(.)\|_{1}=\int_{-1}^{1}|u(t)| d t$. 
For each $x(.) \in W$ define

$$
S_{F, x}:=\left\{f(.) \in L^{1}(I, \mathbb{R}) ; \quad f(t) \in F(t, x(t)) \text { a.e. }(I)\right\} .
$$

LEMMA 2.2. ([1]) If $f():.[-1,1] \rightarrow \mathbb{R}$ is an integrable function and $k \in[-\pi, \pi]$ then the equation

$$
x^{\prime \prime \prime}+k^{2} x^{\prime}=f(t) \quad \text { a.e. }(I)
$$

with the boundary conditions $x(-1)=x(1)=x^{\prime}(1)=0$ has a unique solution given by

$$
x(t)=\int_{-1}^{1} G(t, s) f(s) d s
$$

where $G(.,$.$) is the associated Green function. Namely,$

$$
G(t, x)= \begin{cases}\frac{(1-\cos k(1+x))(1-\cos k(1-t))}{k^{2}(1-\cos 2 k)} & \text { if }-1 \leq x \leq t \leq 1, \\ \frac{(1-\cos k(1+x))(1-\cos k(1-t))-(1-\cos k(x-t))(1-\cos 2 k)}{k^{2}(1-\cos 2 k)} & \text { if }-1 \leq t \leq x \leq 1 .\end{cases}
$$

Moreover, if $k \neq 0$

$$
0 \leq G(t, x) \leq G_{0}:=\frac{k^{2}(5 \sqrt{5}-11)}{\sin ^{2} k} \quad \forall(t, x) \in I \times \mathbb{R} .
$$
on $F$.

In order to study problem (1.1) we introduce the following hypothesis Hypothesis 2.3. (i) $F(.,):. I \times \mathbb{R} \rightarrow \mathcal{P}(\mathbb{R})$ has nonempty closed values and for every $x \in \mathbb{R} F(., x)$ is measurable.

(ii) There exists $L(.) \in L^{\mathbf{1}}\left(I, \mathbb{R}_{+}\right)$such that for almost all $t \in I, F(t, \cdot)$ is $L(t)$-Lipschitz in the sense that

$$
d_{H}(F(t, x), F(t, y)) \leq L(t)|x-y| \quad \forall x, y \in \mathbb{R}
$$

and $d(0, F(t, 0)) \leq L(t)$ a.e. $(I)$.

Denote $L_{0}:=\int_{-1}^{1} L(s) d s$ and assume that $k \neq 0$.

\section{The main results}

We are able now to present a first existence result for problem. (1.1).

Theorem 3.1. Assume that Hypothesis 2.3 is satisfied, $F(.,$.$) has compact$ values and $G_{0} L_{0}<1$. Then the problem (1.1) has a solution.

Proof. We transform the problem (1.1) in a fixed point problem. Consider the set-valued map $T: C(I, \mathbb{R}) \rightarrow \mathcal{P}(C(I, \mathbb{R}))$ defined by

$$
T(x):=\left\{v(.) \in C(I, \mathbb{R}) ; \quad v(t):=\int_{-1}^{1} G(t, s) f(s) d s, \quad f \in S_{F, x}\right\} .
$$


Note that since the set-valued map $F(., x()$.$) is measurable with the mea-$ surable selection theorem (e.g., Theorem III. 6 in [3]) it admits a measurable selection $f():. I \rightarrow \mathbb{R}$. Moreover, from Hypothesis 2.3

$$
|f(t)| \leq L(t)+L(t)|x(t)|,
$$

i.e., $f(.) \in L^{1}(I, \mathbb{R})$. Therefore, $S_{F, x} \neq \emptyset$.

It is clear that the fixed points of $T($.$) are solutions of problem (1.1). We$ shall prove that $T($.$) fulfills the assumptions of Covitz Nadler contraction$ principle.

First, we note that since $S_{F, x} \neq \emptyset, T(x) \neq \emptyset$ for any $x(.) \in C(I, \mathbb{R})$.

Secondly, we prove that $T(x)$ is closed for any $x(.) \in C(I, \mathbb{R})$.

Let $\left\{x_{n}\right\}_{n \geq 0} \in T(x)$ such that $x_{n}(.) \rightarrow x^{*}($.$) in C(I, \mathbb{R})$. Then $x^{*}(.) \in$ $C(I, \mathbb{R})$ and there exists $f_{n} \in S_{F, x}$ such that

$$
x_{n}(t)=\int_{-1}^{1} G(t, s) f_{n}(s) d s .
$$

Since $F(.,$.$) has compact values and Hypothesis 2.3$ is satisfied we may pass to a subsequence (if necessary) to get that $f_{n}($.$) converges to f(.) \in$ $L^{1}(I, \mathbb{R})$ in $L^{1}(I, \mathbb{R})$.

In particular, $f \in S_{F, x}$ and for any $t \in I$ we have

$$
x_{n}(t) \rightarrow x^{*}(t)=\int_{-1}^{1} G(t, s) f(s) d s,
$$

i.e., $x^{*} \in T(x)$ and $T(x)$ is closed.

Finally, we show that $T($.$) is a contraction on C(I, \mathbb{R})$.

Let $x_{1}(),. x_{2}(.) \in C(I, \mathbb{R})$ and $v_{1} \in T\left(x_{1}\right)$. Then there exists $f_{1} \in S_{F, x_{1}}$ such that

$$
v_{1}(t)=\int_{-1}^{1} G(t, s) f_{1}(s) d s, \quad t \in I .
$$

Consider the set-valued map

$$
G(t):=F\left(t, x_{1}(t)\right) \cap\left\{x \in \mathbb{R} ; \quad\left|f_{1}(t)-x\right| \leq L(t)\left|x_{1}(t)-x_{2}(t)\right|\right\}, \quad t \in I .
$$

From Hypothesis 2.3 one has

$$
d_{H}\left(F\left(t, x_{1}(t)\right), F\left(t, x_{2}(t)\right)\right) \leq L(t)\left|x_{1}(t)-x_{2}(t)\right|,
$$

hence $G($.$) has nonempty closed values. Moreover, since G($.$) is measurable,$ there exists $f_{2}($.$) a measurable selection of G($.$) . It follows that f_{2} \in S_{F, x_{2}}$ and for any $t \in I$

$$
\left|f_{1}(t)-f_{2}(t)\right| \leq L(t)\left|x_{1}(t)-x_{2}(t)\right|
$$


Define

$$
v_{2}(t)=\int_{-1}^{1} G(t, s) f_{2}(s) d s, \quad t \in I
$$

and we have

$$
\begin{aligned}
\left|v_{1}(t)-v_{2}(t)\right| & \leq \int_{-1}^{1}|G(t, s)| \cdot\left|f_{1}(s)-f_{2}(s)\right| d s \leq G_{0} \int_{-1}^{1}\left|f_{1}(s)-f_{2}(s)\right| d s \\
& \leq G_{0} \int_{-1}^{1} L(s)\left|x_{1}(s)-x_{2}(s)\right| d s \leq G_{0} L_{0}|| x_{1}-x_{2} \|_{C} .
\end{aligned}
$$

So, $\left\|v_{1}-v_{2}\right\|_{C} \leq G_{0} L_{0}\left\|x_{1}-x_{2}\right\|_{C}$.

From an analogous reasoning by interchanging the roles of $x_{1}$ and $x_{2}$ it follows

$$
d_{H}\left(T\left(x_{1}\right), T\left(x_{2}\right)\right) \leq G_{0} L_{0}\left\|x_{1}-x_{2}\right\|_{C} .
$$

Therefore, $T($.$) admits a fixed point which is a solution to problem (1.1).$

The next theorem is the main result of this paper. As one can see it is, in fact, no necessary to assume that $F(.,$.$) has compact values as in$ Theorem 3.1.

THEOREM 3.2. Assume that Hypothesis 2.3 is satisfied and $G_{0} L_{0}<1$. Let $y(.) \in W$ be such that there exists $q(.) \in L^{1}\left(I, \mathbb{R}_{+}\right)$with

$$
d\left(y^{\prime \prime \prime}(t)+k^{2} y^{\prime}(t), F(t, y(t))\right) \leq q(t) \text { a.e. }(I) .
$$

Then for every $\varepsilon>0$ there exists $x($.$) a solution of problem (1.1) satisfying$ for all $t \in I$

$$
|x(t)-y(t)| \leq \frac{G_{0}}{1-G_{0} L_{0}} \int_{-1}^{1} q(t) d t+\varepsilon .
$$

Proof. For $u(.) \in L^{1}(I, \mathbb{R})$ define the following set valued maps

$$
\begin{gathered}
M_{u}(t)=F\left(t, \int_{-1}^{1} G(t, s) u(s) d s\right), \quad t \in I, \\
T(u)=\left\{\phi(.) \in L^{1}(I, \mathbb{R}) ; \quad \phi(t) \in M_{u}(t) \quad \text { a.e. }(I)\right\} .
\end{gathered}
$$

It follows from Lemma 2.2 that $x($.$) is a solution of problem (1.1) if and$ only if $x^{\prime \prime \prime}()+.k^{2} x^{\prime}($.$) is a fixed point of T($.$) .$

We shall prove first that $T(u)$ is nonempty and closed for every $u \in$ $L^{1}(I, \mathbb{R})$. The fact that the set valued map $M_{u}($.$) is measurable is well$ known. For example the map $t \rightarrow \int_{-1}^{1} G(t, s) u(s) d s$ can be approximated by step functions and we can apply Theorem III. 40 in [3]. Since the values of 
$F$ are closed with the measurable selection theorem (Theorem III.6 in [3]) we infer that $M_{u}($.$) admits a measurable selection \phi$. One has

$$
\begin{aligned}
|\phi(t)| & \leq d(0, F(t, 0))+d_{H}\left(F(t, 0), F\left(t, \int_{-1}^{1} G(t, s) u(s) d s\right)\right) \\
& \leq L(t)\left(1+G_{0} \int_{-1}^{1}|u(s)| d s\right),
\end{aligned}
$$

which shows that $\phi \in L^{1}(I, \mathbb{R})$ and $T(u)$ is nonempty.

On the other hand, the set $T(u)$ is also closed. Indeed, if $\phi_{n} \in T(u)$ and $\left\|\phi_{n}-\phi\right\|_{1} \rightarrow 0$ then we can pass to a subsequence $\phi_{n_{k}}$ such that $\phi_{n_{k}}(t) \rightarrow \phi(t)$ for a.e. $t \in I$, and we find that $\phi \in T(u)$.

We show next that $T($.$) is a contraction on L^{1}(I, \mathbb{R})$.

Let $u, v \in L^{1}(I, \mathbb{R})$ be given and $\phi \in T(u)$. Consider the following set-valued map:

$$
H(t)=M_{v}(t) \cap\left\{x \in \mathbb{R} ; \quad|\phi(t)-x| \leq L(t)\left|\int_{-1}^{1} G(t, s)(u(s)-v(s)) d s\right|\right\} .
$$

From Proposition III.4 in [3], $H($.$) is measurable and from Hypothesis$ 2.3 ii) $H($.$) has nonempty closed values. Therefore, there exists \psi($.$) a mea-$ surable selection of $H($.$) . It follows that \psi \in T(v)$ and according with the definition of the norm we have

$$
\begin{aligned}
\|\phi-\psi\|_{1} & =\int_{-1}^{1}|\phi(t)-\psi(t)| d t \leq \int_{-1}^{1} L(t)\left(\int_{-1}^{1}|G(t, s)| \cdot|u(s)-v(s)| d s\right) d t \\
& =\int_{-1}^{1}\left(\int_{-1}^{1} L(t)|G(t, s)| d t\right)|u(s)-v(s)| d s \leq G_{0} L_{0}\|u-v\|_{1} .
\end{aligned}
$$

We deduce that

$$
d(\phi, T(v)) \leq G_{0} L_{0}\|u-v\|_{1}
$$

Replacing $u$ by $v$ we obtain

$$
d_{H}(T(u), T(v)) \leq G_{0} L_{0}\|u-v\|_{1},
$$

thus $T($.$) is a contraction on L^{1}(I, \mathbb{R})$.

We consider next the following set-valued maps

$$
\begin{gathered}
F_{1}(t, x)=F(t, x)+q(t)[-1,1], \quad(t, x) \in I \times \mathbb{R}, \\
M_{u}^{1}(t)=F_{1}\left(t, \int_{-1}^{1} G(t, s) u(s) d s\right), \\
T_{1}(u)=\left\{\psi(.) \in L^{1}(I, \mathbb{R}) ; \quad \psi(t) \in M_{u}^{1}(t) \quad \text { a.e. }(I)\right\}, \quad u(.) \in L^{1}(I, \mathbb{R}) .
\end{gathered}
$$


Obviously, $F_{1}(.,$.$) satisfies Hypothesis 2.3$.

Repeating the previous step of the proof we obtain that $T_{1}$ is also a $G_{0} L_{0}$-contraction on $L^{1}(I, \mathbb{R})$ with closed nonempty values.

We prove next the following estimate

$$
d_{H}\left(T(u), T_{1}(u)\right) \leq \int_{-1}^{1} q(t) d t
$$

Let $\phi \in T(u)$ and define

$$
H_{1}(t)=M_{u}^{1}(t) \cap\{z \in \mathbb{R} ; \quad|\phi(t)-z| \leq q(t)\} .
$$

With the same arguments used for the set valued map $H($.$) , we deduce$ that $H_{1}($.$) is measurable with nonempty closed values. Hence let \psi($.$) be a$ measurable selection of $H_{1}($.$) . It follows that \psi \in T_{1}(u)$ and one has

$$
\|\phi-\psi\|_{1}=\int_{-1}^{1}|\phi(t)-\psi(t)| d t \leq \int_{-1}^{1} q(t) .
$$

As above we obtain (3.1).

We apply Proposition 2.1 and we infer that

$$
d_{H}\left(\operatorname{Fix}(T), \operatorname{Fix}\left(T_{1}\right)\right) \leq \frac{1}{1-G_{0} L_{0}} \int_{-1}^{1} q(t) d t .
$$

Since $v()=.y^{\prime \prime \prime}()+.k^{2} y^{\prime}(.) \in F i x\left(T_{1}\right)$ it follows that for any $\varepsilon>0$ there exists $u(.) \in \operatorname{Fix}(T)$ such that

$$
\|v-u\|_{1} \leq \frac{1}{1-G_{0} L_{0}} \int_{-1}^{1} q(t) d t+\frac{\varepsilon}{G_{0}} .
$$

We define $x(t)=\int_{-1}^{1} G(t, s) u(s) d s, t \in I$ and we have

$$
|x(t)-y(t)| \leq \int_{-1}^{1}|G(t, s)| \cdot|u(s)-v(s)| d s \leq \frac{G_{0}}{1-G_{0} L_{0}} \int_{-1}^{1} q(t) d t+\varepsilon
$$

which completes the proof.

REMARK 3.3. The assumption in Theorem 3.2 is satisfied, in particular, for $y()=$.0 and therefore, via Hypothesis 2.3 , with $q()=.L($.$) . In this case,$ Theorem 3.1 provides an existence result for problem (1.1) together with a priori bounds for the solution.

\section{References}

[1] G. Bartuzel, A. Fryszkowski, Filippov Lemma for certain differential inclusion of third order, Demonstratio Math. 41 (2008), 337-352. 
[2] A. Bressan, A. Cellina, A. Fryszkowski, A class of absolute retracts in spaces of integrable functions, Proc. Amer. Math. Soc. 112 (1991), 413-418.

[3] C. Castaing, M. Valadier, Convex Analysis and Measurable Multifunctions, Springer, Berlin, 1977.

[4] A. Cernea, Existence for nonconvex integral inclusions via fixed points, Arch. Math. (Brno) 39 (2003), 293-298.

[5] A. Cernea, An existence theorem for some nonconvex hyperbolic differential inclusions, Mathematica (Cluj) 45 (68) (2003), 121-126.

[6] A. Cernea, An existence result for nonlinear integrodifferential inclusions, Comm. Appl. Nonlinear Anal. 14 (2007), 17-24.

[7] A. Cernea, On the existence of solutions for a higher order differential inclusion without convexity, Electron. J. Qual. Theory Differential Equations 8 (2007), 1-8.

[8] A. Cernea, On the existence of mild solutions of a nonconvex evolution inclusion, Math. Commun. 13 (2008), 107-114.

[9] A. Cernea, An existence result for a Fredholm-type integral inclusion, Fixed Point Theory 9 (2008), 441-447.

[10] H. Covitz, S. B. Nadler jr., Multivalued contraction mapping in generalized metric spaces, Israel J. Math. 8 (1970), 5-11.

[11] Z. Kannai, P. Tallos, Stability of solution sets of differential inclusions, Acta Sci. Math. (Szeged) 61 (1995), 197-207.

[12] T. C. Lim, On fixed point stability for set valued contractive mappings with applications to generalized differential equations, J. Math. Anal. Appl. 110 (1985), 436-441.

[13] P. Tallos, A Filippov-Gronwall type inequality in infinite dimensional space, Pure Math. Appl. 5 (1994), 355-362.

\section{FACULTY OF MATHEMATICS AND INFORMATICS}

UNIVERSITY OF BUCHAREST

Academiei 14

010014 BUCHAREST, ROMANIA

E-mail: acernea@fmi.unibuc.ro

Received October 1, 2008; revised version March 25, 2009. 